\title{
$\lambda$ CONNECTIVITY AND MAPPINGS ONTO A CHAINABLE INDECOMPOSABLE CONTINUUM
}

\author{
CHARLES L. HAGOPIAN
}

\begin{abstract}
A continuum (i.e., a compact connected nondegenerate metric space) $M$ is said to be $\lambda$ connected if any two of its points can be joined by a hereditarily decomposable continuum in $M$. Here we prove that a plane continuum is $\lambda$ connected if and only if it cannot be mapped continuously onto Knaster's chainable indecomposable continuum with one endpoint. Recent results involving aposyndesis and decompositions to a simple closed curve are extended to $\lambda$ connected continua.
\end{abstract}

Throughout this paper $D$ will denote Knaster's chainable indecomposable continuum with one endpoint (see [7, p. 332] or [9, Example 1, p. 205]), $I$ will denote the unit interval, and $h$ will denote the function of $I$ onto itself defined by $h(t)=2 t$ for $t \leq 1 / 2$ and $h(t)=2-2 t$ for $t>1 / 2$. D can be represented as an inverse limit of unit intervals, indexed by the positive integers, where the bonding map between successive terms is always $h$.

In $[10], J . W$. Rogers, Jr. proved that every indecomposable continuum can be mapped continuously onto D. Recently D. P. Bellamy [1] generalized this theorem by showing that $D$ is a continuous image of each indecomposable compact connected nondegenerate Hausdorff space. Our principal tool (presented in the following theorem) is derived from Bellamy's proof.

Theorem 1. Suppose that $M$ is a continuum and $\left\{G_{n}\right\}_{n=1}^{\infty}$ is a sequence of nonempty open sets in $M$ such that (1) the closures of $G_{1}$ and $G_{2}$ are disjoint, (2) for each n, $G_{2 n+1} \cup G_{2 n+2} \subset G_{2 n-1}$, and (3) for each $n$, there is a separation $A_{n} \cup B_{n}$ of $M-G_{2 n}$ such that $G_{2 n+1} \subset A_{n}$ and $G_{2 n+2} \subset B_{n}$. Then $M$ can be mapped continuously onto $D$.

Proof. Following Bellamy [1, Theorem (proof)], we let $f_{1}$ be a Urysohn

Presented to the Society, August 23, 1973 under the title $\lambda$ connected continua; received by the editors August 6, 1973.

AMS (MOS) subject classifications (1970). Primary 54C05, 54F 20, 54F 25, 54F60; Secondary 54F 15, 54A05.

Key words and phrases. Hereditarily decomposable continua, $\lambda$ connected continua, Jones' function $K$, arcwise connectivity, aposyndesis, decompositions to a simple closed curve, mappings onto an indecomposable continuum. 
function of $M$ onto $I$ such that $f_{1}\left(G_{1}\right)=0$ and $f_{1}\left(G_{2}\right)=1$. Proceeding inductively, we suppose a continuous function $f_{i}$ of $M$ onto $I$ has been defined for each positive integer $i \leq n$ such that for each $i>1, h \circ f_{i}=f_{i-1}$ and such that for each $i, f_{i}\left(G_{2 i-1}\right)=0$ and $f_{i}\left(G_{2 i}\right)=1$. Define the function $f_{n+1}$ of $M$ onto $I$ by

$$
\begin{aligned}
f_{n+1}(x) & =1 / 2 f_{n}(x) & & \text { if } x \in A_{n}, \\
& =1-1 / 2 f_{n}(x) & & \text { if } x \in B_{n}, \\
& =1 / 2 & & \text { if } x \in G_{2 n} .
\end{aligned}
$$

Note that $f_{n+1}$ is a continuous function, $b \circ f_{n+1}=f_{n}, f_{n+1}\left(G_{2 n+1}\right)=0$, and $f_{n+1}\left(G_{2 n+2}\right)=1$. The sequence $\left\{f_{n}\right\}_{n=1}^{\infty}$ induces a continuous function of $M$ onto $D$.

Theorem 2. A plane continuum $M$ is $\lambda$ connected if and only if $M$ cannot be mapped continuously onto $D$.

Proof. Suppose that $M$ is $\lambda$ connected. In [6], it is proved that every planar continuous image of a $\lambda$ connected continuum is $\lambda$ connected. Note that since $D$ is chainable and indecomposable, it is planar [2, Theorem 4] and not $\lambda$ connected [9, Theorem 7, p. 212]. It follows that $M$ cannot be mapped continuously onto $D$.

To establish the sufficiency part of this theorem we assume that $M$ is not $\lambda$ connected. According to [6, Theorems 1 and 3], there exists an indecomposable continuum $Y$ in $M$ such that every subcontinuum of $M$ that contains a nonempty open subset of $Y$ contains $Y$.

Let $G_{1}$ and $G_{2}$ be open subsets of $M$ that ha ve disjoint closures such that $Y \cap G_{1} \neq \varnothing \neq Y \cap G_{2}$. Proceeding inductively, we assume that open subsets $G_{2 i-1}$ and $G_{2 i}$ of $M$ have been defined for $1 \leq i \leq n$ such that (1) $G_{2 i-1} \cap Y \neq \varnothing \neq G_{2 i} \cap Y$, (2) for each $i<n, G_{2 i+1} \cup G_{2 i+2} \subset G_{2 i-1}$, and (3) for each $i<n$, there is a separation $A_{i} \cup B_{i}$ of $M-G_{2 i}$ such that $G_{2 i+1} \subset A_{i}$ and $G_{2 i+2} \subset B_{i}$. Since every subcontinuum of $M$ that contains $G_{2 n-1} \cap Y$ contains $G_{2 n} \cap Y$, there exist distinct components $W$ and $Z$ of $M-G_{2 n}$ such that $W \cap G_{2 n-1} \cap Y \neq \varnothing \neq Z \cap G_{2 n-1} \cap Y$. Hence there exists a separation $A_{n} \cup B_{n}$ of $M-G_{2 n}$ such that $Y \cap G_{2 n-1} \cap A_{n} \neq \varnothing \neq$ $Y \cap G_{2 n-1} \cap B_{n}$ [9, Theorem 2, p. 169]. Define $G_{2 n+1}$ and $G_{2 n+2}$ to be open subsets of $M$ in $G_{2 n-1} \cap A_{n}$ and $G_{2 n-1} \cap B_{n}$, respectively, such that $G_{2 n+1} \cap Y \neq \varnothing \neq G_{2 n+2} \cap Y$. 
Since the sequence $\left\{G_{n}\right\}_{n=1}^{\infty}$ satisfies the conditions of Theorem 1 , it follows that $D$ is a continuous image of $M$.

Definition. For each set $A$ in a continuum $M$, let $K(A)$ be the intersection of the collection consisting of every continuum in $M$ that contains $A$ in its interior relative to $M$.

This concept is introduced by F. B. Jones [8, Theorem 2]. There the $K$ function is restricted to points (rather than subsets) of a continuum.

Theorem 3. A plane continuum $M$ can be mapped continuously onto $D$ if and only if for some point $x$ of $M$, the set $K(x)$ contains an indecomposable continuum.

Proof. It is known that $M$ is $\lambda$ connected if and only if for each point $x$ of $M$, every continuum in the set $K(x)$ is decomposable [5, Theorem 5]. Hence this theorem follows directly from Theorem 2 .

In [11], H. E. Schlais establishes the following:

Theorem. If $M$ is a hereditarily decomposable continuum, then for each point $x$ of $M$, the interior of $K(x)$ relative to $M$ is void.

E. J. Vought in [12] points out that Schla is' argument [11, Theorem 9 (proof)] also indicates that for each continuum of condensation $H$ in a hereditarily decomposable continuum $M$, the interior of $K(H)$ relative to $M$ is void. Using this fact, Vought then proves the following:

Theorem. Suppose that $M$ is a hereditarily decomposable continuum that is not separated by any of its subcontinua. Then $M$ has a monotone upper semicontinuous decomposition each of whose elements has void interio and whose quotient space is a simple closed curve.

In [4] the author extends Schlais' theorem to $\lambda$ connected plane continua. However, it follows from [3, Theorem 2] and [5, Theorem 1] that every $\lambda$ connected plane continuum that is not separated by any of its subcontinua is hereditarily decomposable. Hence [4] cannot be used to generalize Vought's decomposition theorem.

The following result extends Schlais' theorem to all $\lambda$ connected continua, and leads us to a generalization of Vought's decomposition theorem.

Theorem 4. If $M$ is a $\lambda$ connected continuum, then for each connected nowhere dense subset $H$ of $M$, the interior of $K(H)$ relative to $M$ is void. 
Proof. Assume there exists a connected nowhere dense subset $H$ of $M$ such that the interior of $K(H)$ relative to $M$ is not empty. Let $U$ be a nonempty open subset of $M$ whose closure is contained in the interior of $K(H)$-$H$. Define $L$ to be the component of $M-U$ that contains $H$. Let $Z$ denote the intersection of $L$ and the boundary of $U$. Define $G_{1}$ and $G_{2}$ to be nonempty open subsets of $M$ such that $Z \subset G_{1}, G_{2} \subset U$, and the closures of $G_{1}$ and $G_{2}$ are disjoint subsets of $K(H)-H$. We proceed inductively. Assume that open subsets $G_{2 i-1}$ and $G_{2 i}$ of $M$ have been defined for $1 \leq i \leq n$ such that (1) $Z \subset G_{2 i-1}$, (2) $L$ and the closure of $G_{2 i}$ are disjoint, (3) for each $i<n, G_{2 i+1} \cup G_{2 i+2} \subset G_{2 i-1}$, and (4) for each $i<n$, there is a separation $A_{i} \cup B_{i}$ of $M-G_{2 i}$ such that $G_{2 i+1} \subset A_{i}$ and $G_{2 i+2} \subset B_{i}$.

Note that since $L$ and the closure of $G_{2 n}$ are disjoint, $P=$ (the component of $M-G_{2 n}$ that contains $H$ ) contains $Z$.

Suppose that $P$ contains $G_{2 n-1}$. Since $H$ does not lie in the interior of $P$, there exists a sequence of points $\left\{x_{i}\right\}_{i}^{\infty}=1$ in $M-\left(P \cup G_{2 n}\right)$ converging to a point of $H$. For each positive integer $i$, define $X_{i}$ to be the $x_{i}-$ component of $M-G_{2 n}$. The limit superior $X$ of $\left\{X_{i}\right\}_{i=1}^{\infty}$ is a continuum in $M$ that meets both $H$ and the boundary of $G_{2 n}$ [7, Theorem 2-101, p. 101]. Since $G_{2 n-1} \subset P$, for each positive integer $i, G_{2 n-1} \cap X_{i}=\varnothing$, which implies that $G_{2 n-1} \cap X=\varnothing$. Since $Z \subset G_{2 n-1}$ and $X \cap L \neq \varnothing$, the continuum $X$ does not meet $U$. But this implies that $X$ is a subset of $L$, which contradicts the assumption that $L$ does not meet the closure of $G_{2 n}$. Hence there exists a component $Q$ of $M-G_{2 n}$, distinct from $P$, that intersects $G_{2 n-1}$.

Define $A_{n} \cup B_{n}$ to be a separation of $M-G_{2 n}$ such that $P \subset A_{n}$ and $Q \subset B_{n}$. Let $G_{2 n+1}$ be an open set in $A_{n} \cap G_{2 n-1}$ that contains $Z$. Let $G_{2 n+2}$ be a nonempty open set in $B_{n} \cap G_{2 n-1}$. Note that since $L \subset A_{n}$ and $G_{2 n+2} \subset B_{n}, L$ and the closure of $G_{2 n+2}$ are disjoint.

The sequence $\left\{G_{n}\right\}_{n=1}^{\infty}$ satisfies the conditions of Theorem 1 . Hence the indecomposable plane continuum $D$ is a continuous image of the $\lambda$ connected continuum $M$, which is impossible [6, Theorem 5]. It follows that for each nowhere dense connected subset $H$ of $M$, the interior of $K(H)$ relative to $M$ is void.

Theorem 5. Suppose that $M$ is a $\lambda$ connected continuum that is not separated by any of its subcontinua. Then $M$ has a monotone upper semicontinuous decomposition each of whose elements has void interior and whose quotient space is a simple closed curve. 
Proof. This theorem follows directly from Theorem 4 and [12, Theorem 2 (proof)l.

To see that Theorem 5 actually is a generalization of Vought's theorem, consider the continuum $M$ that is the union of a disk $T$ and a ray that limits on $T$ and has only its endpoint in $T$. The $\lambda$ connected continuum $M$ is not separated by any of its subcontinua. Since $M$ contains a disk, it is not hereditarily decomposable.

\section{REFERENCES}

1. D. P. Bellamy, Composants of Hausdorff indecomposable continua; a mapping approach, Pacific J. Math. 47 (1973), 303-309.

2. R. H. Bing, Snake-like continua, Duke Math. J. 18 (1951), 653-663. MR $13,265$.

3. C. L. Hagopian, Planar images of decomposable continua, Pacific J. Math. 42 (1972), 329-331.

4. - Schlais' theorem extends to $\lambda$ connected plane continua, Proc. Amer. Math. Soc. 40 (1973), 265-267.

5. - Characterizations of $\lambda$ connected plane continua, Pacific J. Math. 49 (1973).

6. - _. $\lambda$ connected plane continua, Trans. Amer. Math. Soc. 191 (1974), 277-287.

7. J. G. Hocking and G. S. Young, Topology, Addison-Wesley, Reading, Mass., 1961. MR 23 \#A2857.

8. F. B、 Jones, Concerning non-aposyndetic continua, Amer. J. Math. 70 (1948), 403-413. MR 9,606.

9. K. Kuratowski, Topology, Vol. 2, 3rd ed., Monografie Mat., Tom 21, PWN, Warsaw, 1961; English transl., Academic Press, New York; PWN, Warsaw, 1968. MR 24 \#A2958; 41 \#4467.

10. J. W. Rogers, Jr., On mapping indecomposable continua onto certain chainable indecomposable continua, Proc. Amer. Math. Soc. 25 (1970), 449-456. MR 41 $\# 1017$.

11. H. E. Schlais, Non-aposyndesis and non-hereditary decomposability, Pacific J. Math. 45 (1973), 643-652.

12. E. J. Vought, Monotone decompositions of continua not separated by any subcontinua, Trans. Amer. Math. Soc. 192 (1974), 67-78.

DEPARTMENT OF MATHEMATICS, CALIFORNIA STATE UNIVERSITY, SACRAME NTO, CALIFORNIA 95819 